Research Article

\title{
Research and Application of Film and Television Literature Recommendation Based on Secure Internet of Things and Machine Learning
}

\author{
Jieqiong Zhou, ${ }^{1,2}$ Zhenhua Wei $\mathbb{D}^{2,3}$ Bin Peng, ${ }^{2,3}$ and Fangchun Chi ${ }^{1}$ \\ ${ }^{1}$ School of Marxism, Xijing University, Xi'an 710123, Shaanxi, China \\ ${ }^{2}$ Graduate School, The University of Perpetual Help System DALTA, Las Piñas Campus, Las Piñas City 1740, Philippines \\ ${ }^{3}$ Faculty of Education, Xi'an Siyuan University, Xi'an 710038, Shaanxi, China
}

Correspondence should be addressed to Zhenhua Wei; james@link.cuhk.edu.hk

Received 14 August 2021; Revised 7 September 2021; Accepted 30 September 2021; Published 15 October 2021

Academic Editor: Sang-Bing Tsai

Copyright ( 2021 Jieqiong Zhou et al. This is an open access article distributed under the Creative Commons Attribution License, which permits unrestricted use, distribution, and reproduction in any medium, provided the original work is properly cited.

Film and television literature recommendation is an AI algorithm that recommends related content according to user preferences and records. The wide application in various APPs and websites provides users with great convenience. This article aims to study the Internet of Things and machine learning technology, combining deep learning, reinforcement learning, and recommendation algorithms, to achieve accurate recommendation of film and television literature. This paper proposes to use the ConvMF-KNN recommendation model to verify and analyze the four models of PMF, ConvM, ConvMF-word2vec, and ConvMF-KNN, respectively, on public datasets. Using the path information between vertices in bipartite graph and considering the degree of vertices, the similarity between items is calculated, and the neighbor item set of items is obtained. The experimental results show that the ConvMF-KNN model combined with the KNN idea effectively improves the recommendation accuracy. Compared with the accuracy of the PMF model on the MovieLens $100 \mathrm{k}$, MovieLens $1 \mathrm{M}$, and AIV datasets, the accuracy of the ConvMF model on the above three datasets is $5.26 \%, 6.31 \%$, and $26.71 \%$, respectively, an increase of $2.26 \%, 1.22 \%$, and $7.96 \%$. This model is of great significance.

\section{Introduction}

With the rapid growth of online film and television literature resources, how to quickly obtain effective information and the latest information from massive amounts of data and information, and how to improve the accuracy and quality of information retrieval to meet the needs of different users for information push, has become an urgent problem to be solved. The diversified information demand is the key to film and television literature recommendation. Generally, users can search for the information they need from massive network resources through information pulling and information pushing. Information pulling means that users actively search for the information resources they need on the Internet according to their own needs. A typical example is a search engine; information push refers to websites, software applications, and so on that push information resources needed by users according to user needs. China's Internet of Things technology started at the same time as other countries in the world, and the Internet of Things industry has developed rapidly in a short time.

With the continuous popularization of film and television software, the amount of information in film and television literature will maintain a rapid growth momentum for a period of time in the future, and the negative impact of information overload will also become more serious. Therefore, the research and application of film and television literature recommendation systems and recommendation algorithms based on the Internet of Things and machine learning have far-reaching significance in resource sharing and solving information overload. 
Compared with the research and application of film and television literature recommendation based on the Internet of Things (IoT) and machine learning, there are many researches on recommendation systems and recommendation algorithms in e-commerce. Therefore, Piao et al. proposed an entropy-based recommendation algorithm to solve the problem of starting the experiment. The results show that this algorithm has high accuracy and is suitable for use. However, the use of this method is more complicated, leading to errors in the results [1]. When implementing security in an IoT network, you will face some problems, such as heterogeneity within the system and the number of devices that need to be resolved. The classification algorithm includes neural network (NN) and support vector machine (SVM), which can be used to detect DoS attacks at the media access control layer. To this end, Canedo and Skjellum used machine learning in IoT gateways to help protect the system, and they studied the use of artificial neural networks (ANNs) in the gateways to detect anomalies in data sent from edge devices. This method can improve the security of the Internet of Things system [2].

The innovation of this paper is as follows. (1) A collaborative filtering algorithm for eye-catching score prediction is proposed to solve the problem of system quality degradation caused by the increase in the number of users. (2) The ConvMF-KNN recommendation model is proposed to recommend film and television literature. Aiming at the influence of noise samples in support vector, this paper proposes a method to deal with noise by using vector criterion in each cluster.

\section{Technology Related to Machine Learning and IoT Security}

2.1. Machine Learning. The neural network and support vector machine training model is based on two variables: conflict rate and arrival rate $[3,4]$. In the neural network, if the possibility of detecting a DoS attack is greater than the set threshold, it is considered that a DoS attack has occurred. The SVM classifies DoS attacks into two levels, low or high, and detects whether an attack has occurred in sequence from low to high [5]. As for the security issues of the Internet of Things, combined with machine learning, the security of the Internet of Things has been greatly improved, effectively reducing failures and preventing privacy leakage. Supervised learning is to infer a model with the ability to identify and judge the future from the labeled training data. Each sample contains an input object and an output object.

2.2. IoT Security. Internet of Things security technology includes several key technologies: network security; identity authorization; encryption; secure side channel attacks; security analysis and threat prediction; interface protection; delivery mechanism; and system development $[6,7]$. The Internet of Things generally consists of three levels: perception layer, network layer, and application layer. As shown in the figure below, the sensing layer is located at the bottom layer, which contains various data collection terminals such as sensors, RFID readers, tags, and cameras [8]. The architecture of the Internet of Things is shown in Figure 1. In the movie recommendation algorithm, based on the collaborative filtering algorithm, this paper uses the idea of content filtering to filter out the movies that are different from users' interests, so as to improve the collaborative filtering and improve the accuracy of movie recommendation.

After collecting various data, it is necessary to implement data classification through a classifier. Different classification algorithms have different accuracy, speed, robustness, etc. The characteristics of the classification algorithm determine the accuracy of the final recommendation. The following introduces three basic classification algorithms and comprehensively compares their performance.

2.2.1. Decision Tree. Decision tree classification algorithm is based on instance samples to classify. It classifies unordered instance samples and infers from them the classification rules in the form of decision tree representation. Through a top-down recursive method, internal nodes (non-leaf nodes) compare attribute values one by one, complete the branch, and finally make a decision at the end of the decision tree (leaf node). The decision tree generation process is shown in Figure 2.

2.2.2. Artificial Neural Network. Artificial neural network (ANN) can abstract, simplify, and simulate the structure and function of the human brain. Artificial neural networks can generally be divided into feedforward networks and feedback networks. In practical applications, the most widely used neural network is the feedforward network, and its network structure is shown in Figure 3.

Although such recommendations may arouse new interests of current users, it is obviously risky to put movies of movie types that users have never ordered in the top position. Therefore, what should be done is to retain but reduce their ranking in the recommendation list. Taking the threelayer BP neural network as an example, the number of neurons in each layer is indeterminate. Assuming that the number of neurons in the input layer, output layer, and hidden layer is $n, \mathrm{~m}$, and $k$ respectively, we get

$$
\begin{aligned}
& h_{i}^{h}(j)=\sum_{i=1}^{n} \omega_{i}^{h} x_{i}(j)-b_{h}, \quad h=1,2, \ldots, k, \\
& h_{l}^{h}(j)=f\left(h_{i}^{h}(j)\right) h=1,2, \ldots, k, \\
& y_{i}^{l}(j)=\sum_{h=1}^{k} \omega_{h}^{l} h_{l}^{h}(j)-b_{l}, \quad l=1,2, \ldots, m, \\
& y_{l}^{l}(j)=f\left(y_{i}^{l}(j)\right) l=1,2, \ldots, m .
\end{aligned}
$$

By decentralizing and increasing transparency and credibility to protect users' privacy and key information, it puts forward a new solution direction for solving the security of the Internet of Things. In the above formula, $x_{i}(j)$ is the $j$-th input 


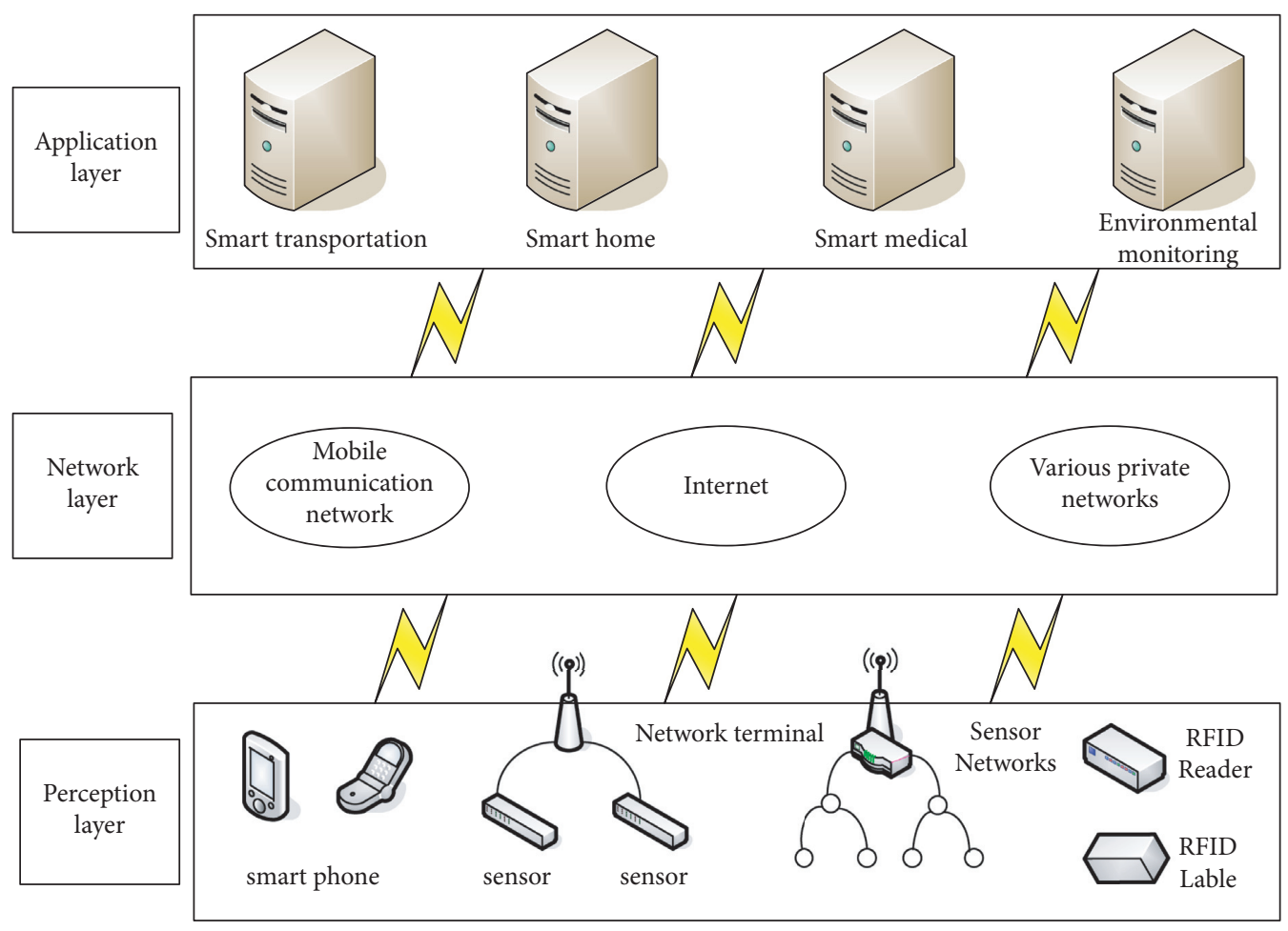

FIgURE 1: IoT architecture.

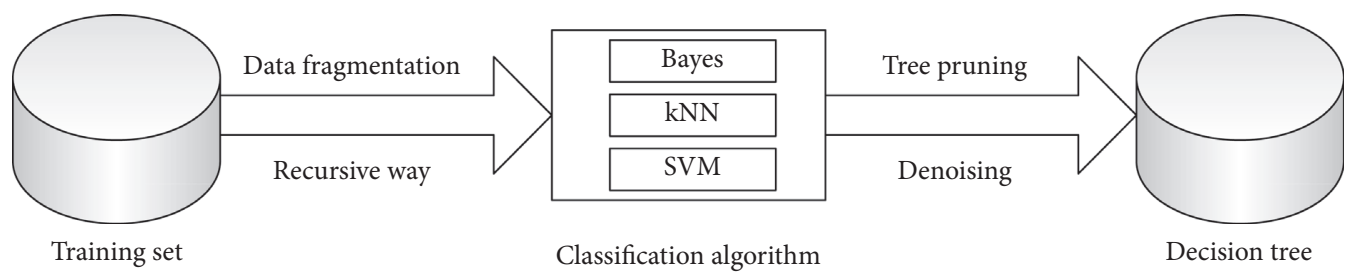

FIgURE 2: Schematic diagram of the decision tree generation process.

sample; in the hidden layer, $h_{i}$ is the input vector and $h_{l}$ is the output vector; in the output layer, $y_{i}$ is the input vector and $y_{l}$ is the output vector; and $f(*)$ is the activation function. The learning and training is to make the error sum of squares reach the minimum, and the error function is

$$
e=\frac{1}{2} \sum_{l=1}^{m}\left(d_{l}(j)-y_{l}^{l}(j)\right)^{2} .
$$

The formula for the global error is as follows:

$$
E=\frac{1}{2 q} \sum_{l=1}^{m}\left(d_{l}(j)-y_{l}^{l}(j)\right)^{2} .
$$

2.2.3. Support Vector Machine. Support vector machine (SVM) is the most commonly used classification algorithm in classification algorithms. It is developed based on the evolution of the optimal classification surface in the case of linear separability. The essence is to divide the points in the space into two categories.
For the linearly separable case, it is necessary to find the classification hyperplane $H$ :

$$
\begin{array}{r}
\omega^{T} x+b=0, \\
y_{i}\left(\omega^{T} x+b\right)-1 \geq 0 .
\end{array}
$$

Then, the $H$ hyperplane can separate the two types of sample points, and the distance formula from any point $x$ to $H$ is

$$
d=\frac{\left|\left(\omega^{T} x+b\right)\right|}{\|\omega\|} .
$$

2.3. Recommendation Algorithm. The memory-based collaborative filtering algorithm has been proposed for a long time and has a long research history. The implementation steps of the algorithm are generally divided into two steps: the first step is to calculate the similarity between the target user and other users and the second step is to find the closest dataset of the target user according to the similarity and then 


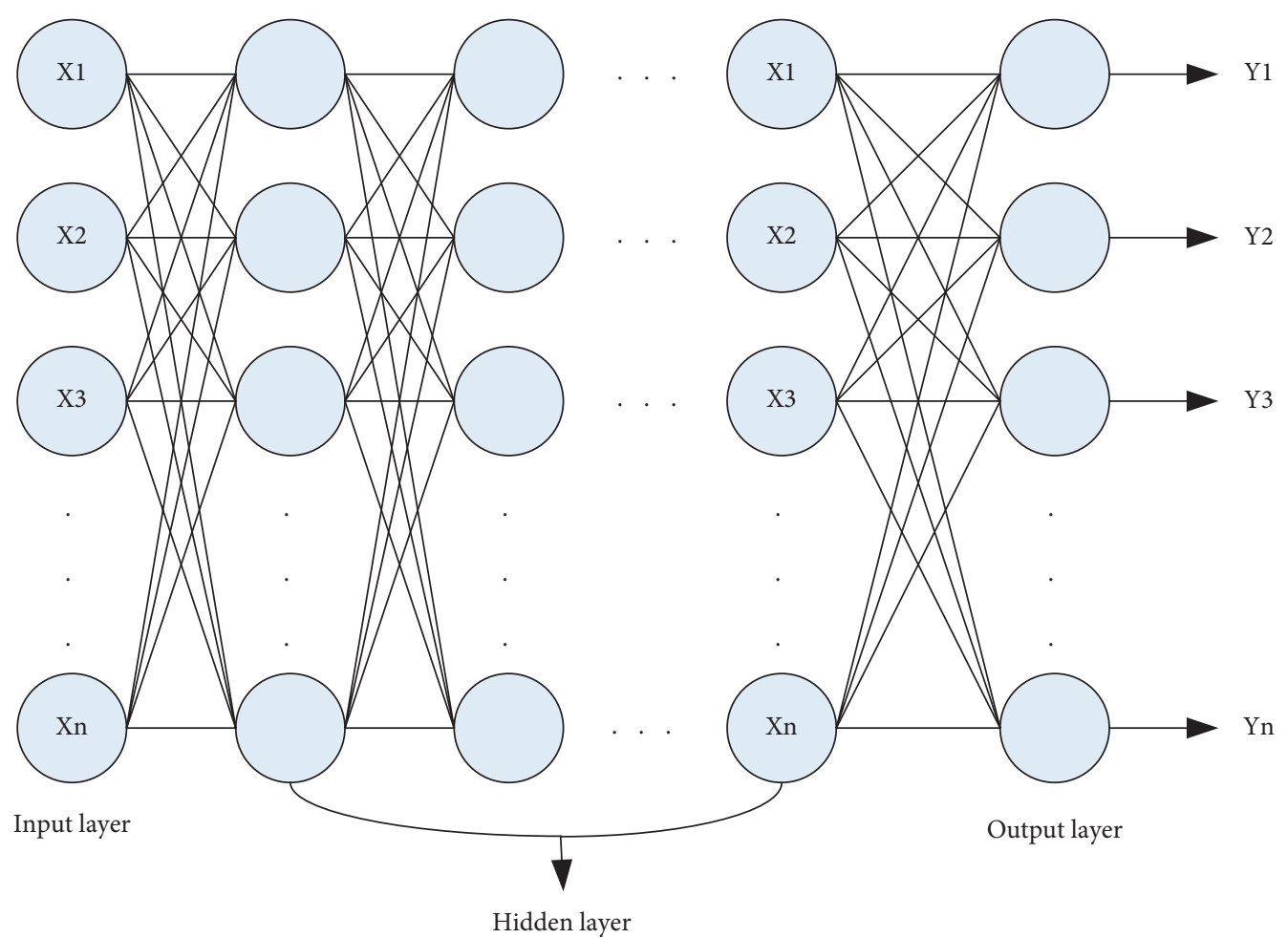

FIgURE 3: Diagram of the decision tree network structure.

use this dataset to predict the target user's score for the scored item [9]. In addition, it can also calculate the similarity between the target item and other items [10]. The classification of recommended algorithms is shown in Figure 4.

Project-based collaborative filtering pays more attention to users' existing hobbies. Generally, movies that are consistent with users' historical preferences are recommended. The movie recommendation algorithm divides the process into three parts, namely, the feature extraction part, the Markov decision process modeling part, and the model training part. The detailed diagram of the movie recommendation algorithm is shown in Figure 5.

The algorithm steps based on the memory algorithm are mainly divided into the following 3 steps.

\subsubsection{Building a User-Item Rating Matrix.} Recommendation systems usually involve two user feedback mechanisms: one is that users rate items, and the system saves the rating information in a relational database. In the $i \times j$ matrix, there are $m$ users and $n$ items. The corresponding value in the matrix is the rating of user $i$ on item $j$, and null means that there is no rating behavior. The other is to collect user behaviors, such as user likes and favorites. When constructing the user-item behavior matrix, this type of behavior is usually expressed in binary. In the $i \times j$ matrix, there are $i$ users and $j$ items, indicating whether user $i$ has performed a certain behavior on item $j ; 0$ indicates that the behavior has not occurred, and 1 indicates that the behavior has occurred.
The matrix composed of ratings between users and items is shown in Table 1, and the matrix composed of ratings between users and behaviors is shown in Table 2 .

2.3.2. Calculating the Similarity between Users and Items. It calculates similar items based on users' interests and preferences. In a memory-based algorithm, it is necessary to calculate the similarity between users and items. Commonly used similarity calculation formulas include the Jaccard similarity coefficient [11], Pearson similarity [12], cosine similarity [13], and so on.

Pearson Similarity. Euclidean distance is a commonly used method in mathematics to find the distance between two points. It can find the absolute distance between two vectors, and it is also a method of calculating similarity. The Euclidean distance is very commonly used in mathematics. The mathematical expression of the Euclidean distance between the vectors $X$ and $Y$ is as follows:

$$
\text { Euclidean distance }(X, Y)=\|X-Y\| \text {. }
$$

Cosine similarity is to calculate the cosine value of the angle between two vectors, which can be used to judge the similarity in the direction of the vectors. If the two vectors are perpendicular, the similarity is 0 ; conversely, if the two vectors are parallel, the similarity is 1 . The mathematical form of the cosine similarity of two vectors $M$ and $N$ is defined as follows: 


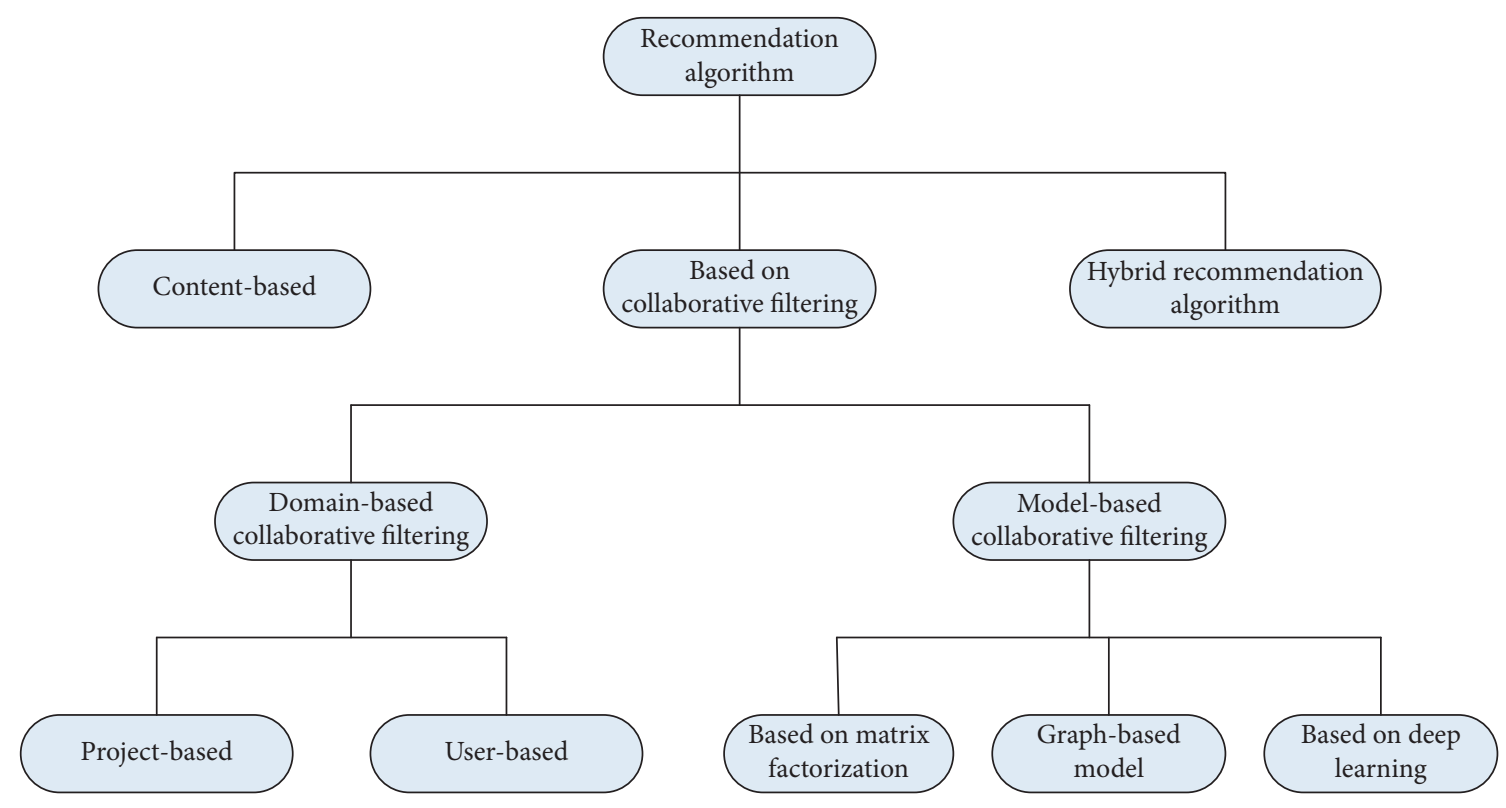

FIGURE 4: Recommended algorithm classification.

$$
\cos \theta(M, N)=\frac{M \cdot N}{\|M\| N \|}
$$

Pearson similarity is to calculate the relative distance between the two. The value range of Pearson similarity is $[-1$, +1 ], -1 means dissimilar, +1 means similar, and the range from -1 to +1 means similarity. The degree of dissimilarity or similarity between the two is as follows:

$$
\operatorname{sr}(m, n)=\frac{\sum_{u \in U_{m n}}\left(r_{u m}-\overline{r_{m}}\right)\left(r_{u n}-\overline{r_{n}}\right)}{\sum_{u \in U_{m n}}\left(r_{u m}-\overline{r_{m}}\right)^{2} \sqrt{\sum_{u \in U_{m n}}\left(r_{u n}-\overline{r_{n}}\right)^{2}}}
$$

where sr stands for similar, which represents the degree of similarity between items, $r_{u m}$ and $r_{u n}$ represent user u's ratings of items $m$ and $n$, respectively, $U_{m n}$ represents users who have rated both items $m$ and $n, \overline{r_{m}}$ represents the average score of users in $U_{m n}$ for item $m$, and $\bar{r}_{n}$ represents the average score of users in $U_{m n}$ for item $n$.

Jaccard Coefficient. It is equal to the ratio of the number of intersections of the sample set to the number of unions and is used to measure the similarity of two sets. The calculation of this index is shown in formula (4).

$$
\operatorname{sr}(i, j)=\frac{N(i) \cap N(j)}{N(i) \cup N(j)}
$$

where $\operatorname{sr}(i, j)$ represents the similarity between user $i$ and user $j$ and $N(i)$ and $N(j)$, respectively, represent the number of items rated by user $i$ and user $j$ [14].
Cosine Similarity. It judges similarity by calculating the cosine value of the angle between two vector dot product spaces, and its cosine value range is $[-1,1]$. The key to cosine similarity is to look at the difference in vector direction, not the difference in length or distance [15]. The calculation of this index is shown in the following formula:

$$
\operatorname{sr}(m, n)=\frac{\sum_{u \in U_{m n}} r_{u m} r_{u n}}{\sqrt{\sum_{u \in U_{m}}\left(r_{u m}\right)^{2}} \sqrt{\sum_{u \in U_{n}}\left(r_{u n}\right)^{2}}} .
$$

After calculating the similarity of the aforementioned scoring matrix or behavior matrix, the data are screened according to the similarity to find the nearest neighbor set of the target item or user. There are generally two methods for screening and generating the nearest neighbor set: one is the nearest neighbor classification algorithm [16] and the other is the threshold method [17]. In the nearest neighbor classification algorithm, the predicted data should be compared with the training samples to find the most similar $\mathrm{K}$ training samples, and the label that appears the most in these K training samples is used as the final prediction label. The commonly used algorithm is mainly the KNN algorithm.

2.3.3. Generating Recommendation Results Based on the Nearest Neighbor Set. After generating the nearest neighbor set, we can predict the ratings of other unrated items and movies based on the target user's ratings of items and movies, then select the top $\mathrm{N}$ movies with the highest predicted scores, and recommend them to the users. The prediction scoring formula is as follows: 


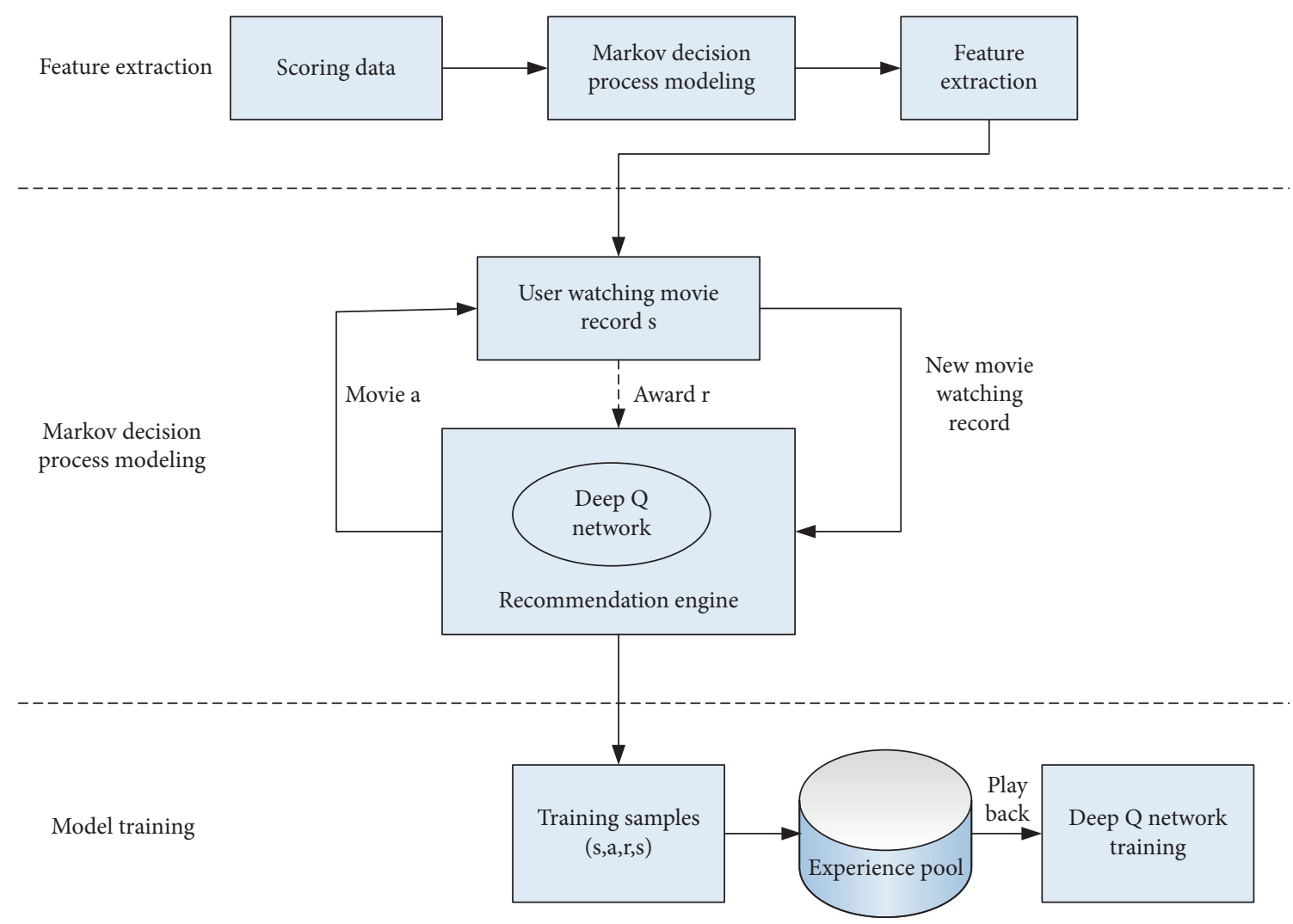

FIGURE 5: Schematic diagram of movie recommendation algorithm.

$$
\begin{aligned}
Q_{u x} & =\frac{\sum_{y \in N} \operatorname{sr}(x, y)\left(r_{u x}\right)}{\sum_{y \in N} \operatorname{sr}(x, y)}, \\
Q_{u x} & =\overline{r_{x}}+\frac{\sum_{y \in N} \operatorname{sr}(x, y)\left(r_{u x}-\overline{r_{y}}\right)}{\sum_{y \in N} \operatorname{sr}(x, y)} .
\end{aligned}
$$

\section{Experimental Design}

3.1. Data Preprocessing. The film introduction information contains a large number of invalid information such as punctuation marks and spaces, which cannot be used as the input of the CNN structure. Therefore, it is necessary to preprocess the movie introduction information. First, remove the punctuation marks, spaces, prepositions, and other characters in the movie information. The maximum number of all words in each profile is max_length. If the length exceeds this length, it will be truncated directly, and only the words before max_length in the profile will be processed. Then, put all the words introduced in the movie into a list $L$, sort the words from high to low, and select the vocab_size words with the highest frequency and not exceed the threshold max_df to form the list Voc. Perform word vector transformation on the words in $L$. For words that appear in $L$ but not in Voc, the average value of all word vectors in Voc is used for word vectorization. It simulates the processing method of biological neurons. When the signal reaches a certain condition after processing, it transmits the signal to the next layer.

3.2. CNN Model and Hyperparameters. The specific design of $\mathrm{CNN}$ network structure is shown in Figure 6. Figure 6 details the design and specific configuration of each layer in the CNN network structure. This paper optimizes the recommendation system based on collaborative filtering to make the recommendation system more personalized and accurate.

The convolution layer contains three convolution kernels with sizes of $300 * 3,300 * 4$, and $300 * 5$. The input of the convolutional layer is the output of the embedding layer. After each convolutional layer, there is a pooling layer, and the three pooling layers get output results of size 100, respectively. Connect these three pooling layers in parallel as the input of the fully connected layer 1 . Each model has a corresponding hyperparameter, and each hyperparameter will have a different impact on the accuracy of the model. Especially in deep learning technology, parameter training and selection are extremely important and require a lot of time and energy. 
TABLE 1: User-item rating matrix.

\begin{tabular}{lcccc}
\hline & Item $_{1}$ & Item $_{2}$ & $\ldots$ & \\
\hline User $_{1}$ & $R_{11}$ & null & $\ldots$ & \\
User $_{2}$ & null & $R_{22}$ & $\ldots$ & null \\
$\ldots$ & $\ldots$ & $\ldots$ & $\ldots$ & null \\
User $_{j}$ & $R_{11}$ & $R_{11}$ & $\ldots$ & $\ldots$ \\
\hline
\end{tabular}

TABLE 2: User-item behavior matrix.

\begin{tabular}{lcccc}
\hline & Item $_{1}$ & Item $_{2}$ & $\ldots$ & \\
\hline User $_{1}$ & 1 & 1 & $\ldots$ & Item $_{i}$ \\
User $_{2}$ & 1 & 1 & $\ldots$ & 0 \\
$\ldots$ & $\ldots$ & $\ldots$ & $\ldots$ & $\ldots$ \\
User $_{j}$ & 1 & 0 & $\ldots$ & $\ldots$ \\
\hline
\end{tabular}

3.3. ConvMF-Word2vec. The original data are randomly shuffled and then sliced, divided into training set, verification set, and test set according to $8: 1: 1$. The training set is used to train the ConvMF-word2vec model, and the validation set is used to verify the results of the trained model. Finally, the performance of the ConvMF-word2vec model is tested on the test set to obtain the final RMSE. The Google Engine database training set is used as the initial embedding layer of $\mathrm{CNN}$ in the ConvMF-word2vec experimental process.

3.4. ConvMF-KNN. The original data are randomly shuffled and then sliced, divided into training set, verification set, and test set according to $8: 1: 1$. Train the ConvMF-KNN model on the training set, verify the model obtained after training on the training set in the validation set, and finally judge the performance of the ConvMF-KNN model on the test set to get the final RMSE. In the ConvMF-KNN experiment process, $\mathrm{KNN}$ is used to correct each scoring item predicted by ConvMF.

\section{Experimental Results and Analysis}

4.1. Experimental Results and Analysis of ConvMF on MovieLens $100 \mathrm{~K}$. Under the premise of traditional passive intrusion detection system, the received signal strength of each node in the network is used to detect the intrusion signal. In order to optimize the ConvMF model, through grid search, on the MovieLines $100 \mathrm{~K}$ dataset, when the learning rate $=0.00001, \mathrm{D}=50,=100,=1$, ConvMF at this time RMSE obtains the minimum value of 0.9258 . The personalized movie recommendation system is usually active recommendation. Through the analysis of users' information and behavior, it can mine users' preferences and then recommend movies to users. Specifically, the impact of RMSE on MovieLens $100 \mathrm{~K}$ is shown in Table 3.

4.2. Experimental Results and Analysis of ConvMF-KNN on MovieLens $100 \mathrm{~K}$. On the dataset ml-100k, the recommended accuracy (RMSE) of ConvMF-KNN is affected by the value of $k$. It can be seen from Figure 7 that when the value of $k$ gradually increases from 10, the RMSE1 gradually decreases. When $K=100$, the RMSE reaches the minimum value, and the recommended accuracy is the most accurate at this time. When the value of $k$ is greater than 100 , there is no significant change in RMSE1. From the dataset, we can calculate that the average number of movies rated by each user is about 100 . Therefore, when $K=100$, the recommendation accuracy is the most accurate, which means that the movies watched by each user have been fully considered. When $K$ is greater than 100, RMSE1 obviously changes very little, as shown in Figure 7. Contentbased recommendation algorithm mainly uses content filtering technology to filter out items similar to users' interests in a large number of items, so as to recommend them to users.

4.3. The Improved Model and the Experimental Results and Analysis of PMF and ConvMF on the Datasets MovieLens $100 \mathrm{~K}$ and MovieLens $1 \mathrm{M}$. The comparison of the experimental results of each model is shown in Table 4.

Explanation and analysis of Table 4:

(1) The improved models ConvMF-word2vec and ConvMF-KNN have improved accuracy on the datasets MovieLens $100 \mathrm{k}$, MovieLens $1 \mathrm{M}$, and AIV, respectively.

(2) After ConvMF-word2vec uses the Google engine database pretraining vector set, the recommendation accuracy on the MovieLens $100 \mathrm{k}$ and MovieLens $1 \mathrm{M}$ data sets is slightly improved compared to ConvMF, but the effect is not obvious, only about $1 \%$.

(3) The ConvMF-KNN model combined with the KNN idea has a greater impact on the recommended accuracy. The accuracy of the PMF model on the MovieLens 100k, MovieLens 1M, and AIV datasets has increased by $5.26 \%, 6.31 \%$, and $26.71 \%$, respectively. Compared with the ConvMF model, its accuracy has increased by $2.26 \%, 1.22 \%$, and $7.96 \%$, respectively. 


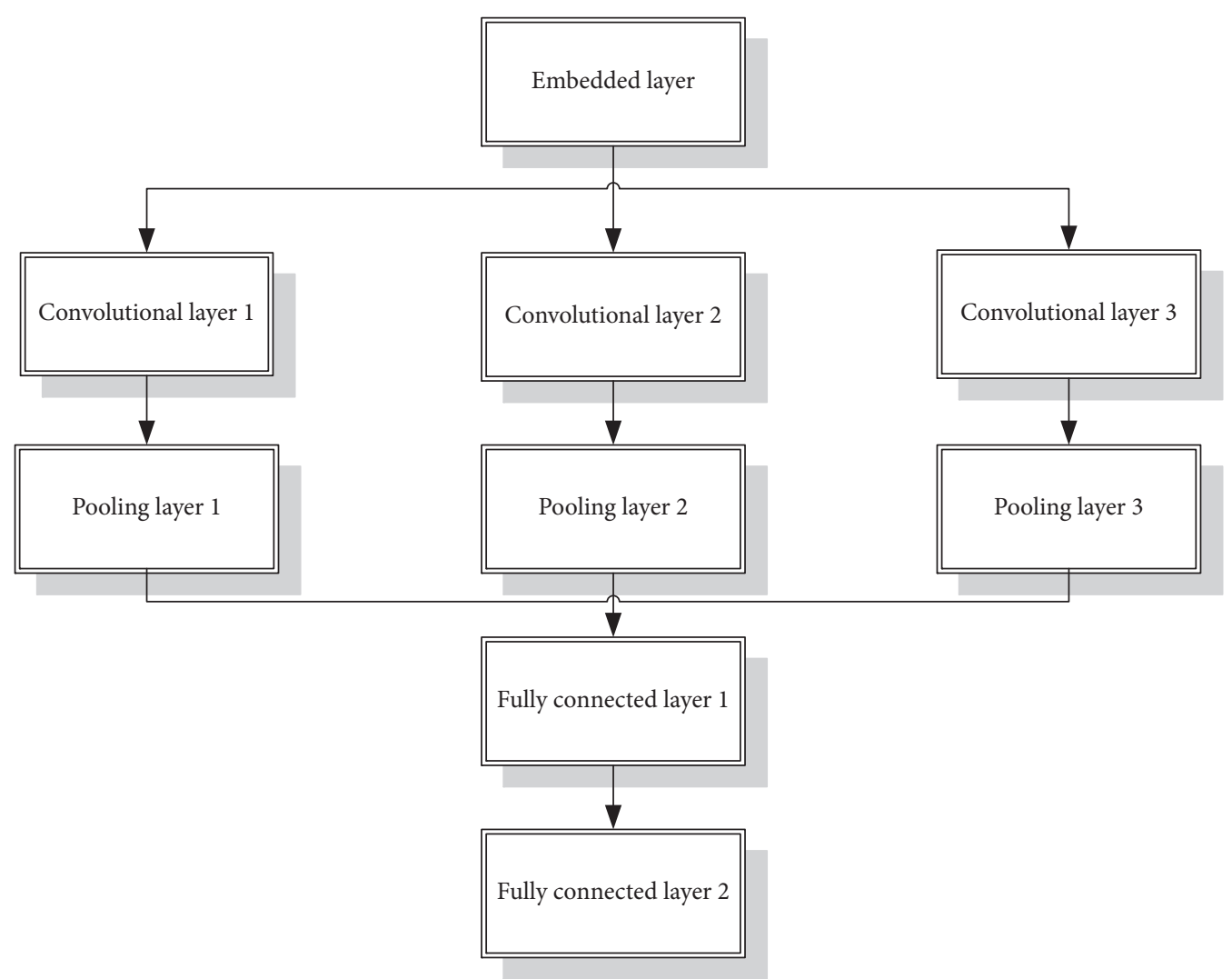

Figure 6: CNN structure's specific design drawing.

TABle 3: ConvMF vs. MovieLens 100K RMSE comparison.

\begin{tabular}{lcccc}
\hline & $\alpha_{u}=1$ & $\alpha_{u}=10$ & $\alpha_{u}=100$ & $\alpha_{u}=100$ \\
\hline$\alpha_{v}=1$ & 1.2735 & 1.0402 & 0.9503 & 0.9293 \\
$\alpha_{v}=10$ & 0.9949 & 0.9542 & 0.9265 & 0.9957 \\
$\alpha_{v}=100$ & $\mathbf{0 . 9 2 5 8}$ & 0.9274 & 0.9945 & 1.2092 \\
$\alpha_{v}=1000$ & 1.0193 & 1.1771 & 1.3723 & 1.9811 \\
\hline
\end{tabular}

Bold values represent the minimum value.

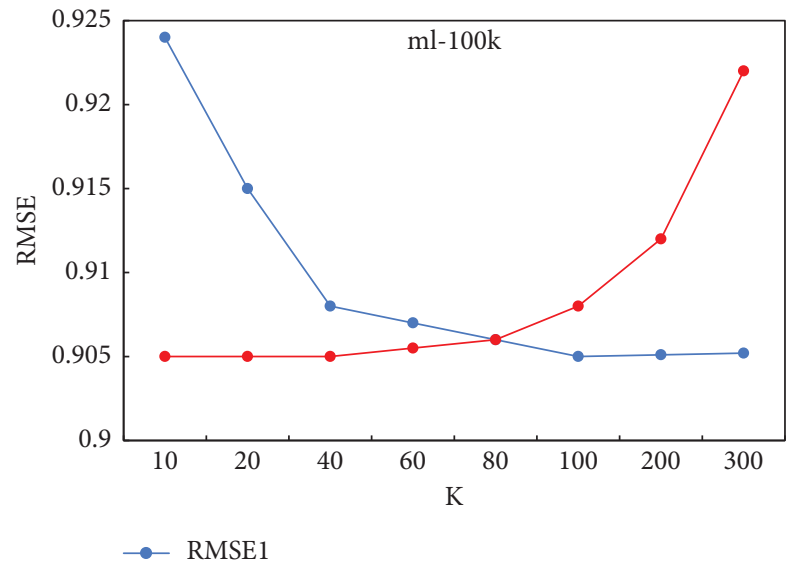

Figure 7: Experimental results of ConvMF-KNN on MovieLens 100K. 
TABLE 4: Comparison of RMSE results of each model.

\begin{tabular}{lccr}
\hline & MovieLens 100k & MovieLens 1 M & AIV \\
\hline PMF & 0.9522 & 0.8962 & 1.4253 \\
ConvMF & 0.9248 & 0.8513 & 1.1343 \\
ConvMF-word2vec & 0.9212 & 0.8562 & 1.1256 \\
ConvMF-KNN & 0.9038 & 0.8472 & 1.0427 \\
\hline
\end{tabular}

\section{Conclusions}

The Internet of Things security technology, machine learning, and recommendation algorithms are developing rapidly, and the recommendation of film and television literature is becoming more and more popular. People generally start to look for movies and literature they like on the Internet. The film industry has been developing for many years, a large number of films already exist in the world, and a large number of new films will be released every year, and the phenomenon of film information overload will become more and more serious. In the face of massive amounts of videos, the application of recommendation algorithms to the film and video industry has improved the user experience. The classic collaborative filtering algorithm has played an irreplaceable role, but with the development of the times and the maturity of deep learning and other technologies, traditional recommendation algorithms are gradually combined with deep learning and other technologies, and a series of new algorithms are gradually produced. This article introduces common recommendation algorithms and focuses on the recommendation algorithm based on scoring matrix and the probability matrix factorization recommendation algorithm and a recommendation algorithm based on deep learning, ConvMF, combined with the idea of KNN to improve the ConvMF algorithm. A new algorithm, ConvMF-KNN, improves the recommendation accuracy of the dataset. The application of recommendation algorithms in film and television literature will become increasingly widespread.

\section{Data Availability}

The data that support the findings of this study are available from the corresponding author upon reasonable request.

\section{Conflicts of Interest}

The authors declare that they have no potential conflicts of interest with respect to the research, authorship, and/or publication of this article.

\section{Acknowledgments}

This study was supported by Educational Research Fund (SGH20Y1529) of a Study on the Teaching Status of Humanities General Education Courses in Private Universities and Reform Strategies: A Case Study of Five Private Universities in Shaanxi. This study was also supported by Educational Research Fund (YBKT-1801) of Shaanxi Education Bureau.

\section{References}

[1] C. H. Piao, Z. Jing, and L. J. Zheng, "Research on entropybased collaborative filtering algorithm and personalized recommendation in e-commerce," Service Oriented Computing and Applications, vol. 3, no. 2, pp. 147-157, 2019.

[2] J. Canedo and A. Skjellum, "Using machine learning to secure IoT systems," in Proceedings of the 2016 14th Annual Conference on Privacy, Security and Trust (PST), vol. 9, no. 4, pp. 346-352, IEEE, Auckland, New Zealand, 2016.

[3] A. L. De Ng, Y. Y. Zhu, and B. L. Shi, "A collaborative filtering recommendation algorithm based on item rating prediction," Journal of Software, vol. 14, no. 9, pp. 54-65, 2013.

[4] S. Li, D. X. Li, and S. Zhao, "The internet of things: a survey," Information Systems Frontiers, vol. 17, no. 2, pp. 243-259, 2015.

[5] A. Al-Fuqaha, M. Guizani, M. Mohammadi, M. Aledhari, and M. Ayyash, "Internet of things: a survey on enabling technologies, protocols, and applications," IEEE Communications Surveys \& Tutorials, vol. 17, no. 4, pp. 2347-2376, 2015.

[6] N. Ramu, V. Pandi, J. D. Lazarus, and S. Radhakrishnan, "A novel trust model for secure group communication in distributed computing," Cloud Computing, vol. 32, no. 3, 2020.

[7] S. S. Gill and A. Shaghaghi, "Security-aware autonomic allocation of cloud resources: a model, research trends, and future directions," Cloud Computing, vol. 32, no. 3, 2020.

[8] Y. Liu, Extremal Entropy: Information Geometry, Numerical Entropy Mapping, and Machine Learning Application of Associated Conditional Independences, Drexel University, vol. 17, no. 2, pp. 245-258, Philadelphia, PA, USA, 2016.

[9] J. P. Cohen, Automated Crater Detection using Machine Learning, vol. 15, no. 2, pp. 178-185, 2016.

[10] S. Qi, C. Hu, K. Hu, J. Xie, and P. Fang, "Research and reflection on recommendation system based on machine learning and TCM theory," Jiangsu Science \& Technology Information, vol. 9, no. 3, pp. 241-245, 2017.

[11] M. Ummesalma and C. Yashiga, "COLPOUSIT: a hybrid model for tourist place recommendation based on machine learning algorithms," in Proceedings of the 2021 5th International Conference on Trends in Electronics and Informatics (ICOEI), vol. 11, no. 4, pp. 170-175, Tirunelveli, India, 2021.

[12] W. Liu and X. Wenfeng, "Application of intelligent recommendation technology based on machine learning in transformer selection," Electric Power Information and Communication Technology, vol. 12, no. 1, pp. 255-268, 2019.

[13] H. Cong, "Personalized recommendation of film and television culture based on an intelligent classification algorithm," Personal and Ubiquitous Computing, vol. 24, no. 2, pp. 165-176, 2020.

[14] Z. Cai, C. Zhou, and X. Li, Application Research of Employment Recommendation Based on Improved K-Means Algorithm in Colleges and Universities, Springer, vol. 14, no. 2, pp. 358-369, Cham, Switzerland, 2019.

[15] L. Chunhua, "Research on the influence of new media on the creation of drama and film literature," International Society of 
Computer Science and Electronic Technology, vol. 15, no. 3, pp. 456-462, 2019.

[16] Z. Nan, "Research on text recognition method based on artificial intelligence," Machine Learning, vol. 16, no. 2, pp. 365-372, 2016.

[17] H. K. Han and E. H. Suh, "Recommendation system for students' learning strategies based on machine learning," International Conference on Future Information \& Communication Engineering, vol. 9, no. 1, pp. 73-75, 2017. 